\title{
Management of glioblastoma: an Australian perspective
}

\author{
Hao-Wen Sim ${ }^{1,2,3,4}$, Anna K. Nowak ${ }^{5,6}$, Zarnie Lwin ${ }^{7,8}$, Mustafa Khasraw ${ }^{1,9}$ \\ ${ }^{1}$ NHMRC Clinical Trials Centre, University of Sydney, Sydney, NSW, Australia; ${ }^{2}$ St Vincent's Clinical School, University of New South Wales, \\ Sydney, NSW, Australia; ${ }^{3}$ Department of Medical Oncology, The Kinghorn Cancer Centre, Sydney, NSW, Australia; ${ }^{4}$ Department of Medical \\ Oncology, Chris O’Brien Lifehouse, Sydney, NSW, Australia; ${ }^{5}$ Medical School, University of Western Australia, Perth, WA, Australia; ${ }^{6}$ Department \\ of Medical Oncology, Sir Charles Gairdner Hospital, Perth, WA, Australia; ${ }^{7}$ School of Medicine, University of Queensland, Brisbane, QLD, \\ Australia; ${ }^{8}$ Department of Medical Oncology, Royal Brisbane and Women's Hospital, Brisbane, QLD, Australia; ${ }^{9}$ The Preston Robert Tisch Brain \\ Tumor Center and Duke Center for Cancer Immunotherapy, Duke University, Durham, NC, USA \\ Correspondence to: Hao-Wen Sim. NHMRC Clinical Trials Centre, University of Sydney, ABN 15211 513 464, Locked Bag 77, Camperdown, \\ Sydney, NSW 1450, Australia. Email: hao-wen.sim@ctc.usyd.edu.au.
}

Submitted Jan 10, 2020. Accepted for publication Feb 03, 2020.

doi: $10.21037 /$ cco.2020.02.05

View this article at: http://dx.doi.org/10.21037/cco.2020.02.05

\section{Introduction}

Although glioblastoma is a rare cancer, it causes disproportionately high morbidity and mortality. In addition to the upfront challenges of neurosurgical resection, radiotherapy and chemotherapy, there are complex care issues including physical disability, cognitive impairment, personality change, depression, seizures and caregiver distress (1).

Comprehensive multidisciplinary care for glioblastoma is essential, including neurosurgery, radiation oncology, medical oncology, neurology, palliative care, neurological rehabilitation, social work, neuropsychology and psychiatry. However, the optimal delivery of care is contingent on available specialty services, local infrastructure and resources. This is pertinent to a sparsely populated country such as Australia, where access to healthcare may vary between metropolitan, regional and rural centers.

We describe the contemporary management of glioblastoma in Australia, focusing on key epidemiology, clinical management, insurance landscape, clinical trials and future directions applicable to the Australian setting.

\section{Epidemiology}

In Australia, the age-adjusted incidence rate of primary brain cancer is 6.9 per 100,000 , which makes it the $18^{\text {th }}$ most common cancer (2). Incidence increases with age, but there is a relative peak in childhood and adolescence; from 15 to 24 years, primary brain cancer is the $2^{\text {nd }}$ most common cancer after leukemia. By gender, the incidence of primary brain cancer is similar during childhood, but diverges with age leading to a higher incidence in men than women overall. Although information specific to glioblastoma is not available in detail annually, data from 2013 record 982 new cases of glioblastoma in Australia, constituting 62\% of all neuroepithelial tumors (3). Of these, $58 \%$ were in males, and median age at diagnosis was approximately 65 years.

Globally, there is widespread geographic variation in the incidence of primary brain cancer, being highest in central and western Europe and lowest in Africa (4). For example, age-adjusted incidence rates in Iceland, Denmark and Norway are 20.8, 19.4 and 17.3 per 100,000 respectively, which are at least twice that of Australia. The United States epidemiology is similar to Australia, with an age-adjusted incidence rate of 7.1 per 100,000 (5). This incidence pattern is independent of socio-demographic index, and age and gender distributions are similar. The reasons for geographic variation remain unclear, but supports possible genetic susceptibility and environmental factors. Notably, the cause of glioblastoma is usually unknown, with known risk factors including ionizing radiation exposure and rare familial syndromes $(6,7)$.

In Australia, the age-adjusted mortality rate of primary brain cancer is 5.3 per 100,000 , making it the $11^{\text {th }}$ leading cause of cancer-related death (2). Due to the predilection in childhood, it is the leading cause of cancer-related death in those under 15 , and the $2^{\text {nd }}$ leading cause of cancerrelated death in the 15 to 24 years age group. The 5 -year relative survival for primary brain cancer is $22.1 \%$, without 
appreciable improvement over 30 years. This is slightly worse than the United States, where the age-adjusted mortality rate of primary brain cancer is 4.4 per 100,000 and 5 -year relative survival is $35.8 \%$ (5). For glioblastoma specifically, the 5 -year relative survival is $4.6 \%$ in Australia vs. $6.8 \%$ in the United States $(3,5)$.

Regarding regional variation, Australia is one of the most sparsely populated countries in the world (8). Of the Australian population, $72 \%$ reside in major cities, $26 \%$ in regional areas and $2 \%$ in remote areas. Overall, cancer survival in Australia was highest in major cities, followed by regional and then remote areas. Based on New South Wales state data, the relative excess risk of death from cancer in remote areas was 1.4 times that of highly accessible areas, with the greatest disparities being for prostate and cervical cancer (9). Similarly, based on Queensland state data, the relative excess risk of death from cancer in remote areas was 1.3 and 1.2 times that of the Queensland average, for men and women respectively (10). A potential explanation is that those living in rural areas may have poorer access to cancer services and may delay seeking medical attention.

However, this regional trend was not evident for primary brain cancer. In New South Wales, the relative excess risk of death from primary brain cancer in accessible, moderately accessible and remote areas was 1.0, 1.1 and 0.3 times that of highly accessible areas, respectively (9). In Queensland, there was no evidence of geographical variation in the relative excess risk of death from primary brain cancer $(\mathrm{P}=0.99$ in men and $\mathrm{P}>0.999$ in women) $(10)$. This distinction may reflect the prevailing neurosurgical referral pathways in Australia, which centralize the care of primary brain cancer patients within subspecialty cancer centers in major cities.

\section{Clinical management}

The Australian healthcare model is two-tiered, consisting of public universal healthcare and optional private health insurance. The majority of patients with glioblastoma are managed in the public sector. The initial treatment of glioblastoma closely adheres to the Stupp protocol, which is a combined modality strategy consisting of maximal safe neurosurgery, then radiotherapy with concomitant and adjuvant temozolomide (11). Neurosurgery is typically performed by subspecialist brain tumor surgeons in major cities. Patients presenting to emergency departments of regional and rural areas are transferred to major cities for further workup using established referral pathways.
Given the subspecialist setting, there is frequently access to neuro-navigation, intraoperative monitoring, use of awake craniotomy, intraoperative magnetic resonance imaging (MRI) and fluorescent agents such as 5-aminolevulinic acid $\left(\right.$ Gliolan $\left.^{\circledR}\right)$, as indicated. It is notable that the adjunctive use of carmustine wafer implants $\left(\right.$ Gliadel $\left.^{\circledR}\right)$ is limited in Australia, due to wariness about risk of surgical site infection, inability to access clinical trials after use of Gliadel $^{\circledR}$ and lack of government reimbursement for temozolomide after use of Gliadel ${ }^{\circledR}$ (12).

Following neurosurgery, most cases are presented at neuro-oncology multidisciplinary meetings, which are convened in about $81 \%$ of subspecialty cancer centers (11). This facilitates review of the integrated diagnosis of glioblastoma and treatment planning, as well as discussion of subsequent management dilemmas such as suspected pseudoprogression, the role of repeat resection and/or reirradiation at relapse.

Adjuvant radiotherapy for primary brain cancer is predominantly administered in major cities, albeit radiotherapy centers have recently been established in larger regional areas as part of a government initiative to improve cancer care access for regional and rural Australians (11). For those who need to travel from afar, subsidized patient accommodation is usually available near the treating center. Radiotherapy centers in Australia are equipped to deliver photon therapy using three-dimensional conformal, intensity-modulated and/or stereotactic techniques. Proton beam therapy is currently unavailable in Australia; regardless, there is insufficient evidence that it should be used for glioblastoma. The first proton treatment unit is under construction in Adelaide, South Australia and due for completion in 2022.

Most glioblastoma patients in Australia will receive long-course radiotherapy consisting of 60 Gray in 30 fractions with concomitant and adjuvant temozolomide, including fit patients aged over 70 . There is increasing acceptance of alternative treatment regimens for elderly or frail patients, including short-course radiotherapy consisting of 40 Gray in 15 fractions with concomitant and adjuvant temozolomide, short-course radiotherapy alone for $\mathrm{O}^{6}$-methylguanine DNA-methyltransferase (MGMT)unmethylated glioblastoma, or temozolomide monotherapy for MGMT-methylated glioblastoma (13-15). Regarding the adjunctive use of alternating electric field therapy such as tumor treating fields (TTF), TTF is not a registered device in Australia, thus patients must travel overseas in order to access it. Furthermore, there is controversy 
about the comparative cost-effectiveness of TTF for the Australian healthcare setting (16).

The mainstay of systemic therapy for glioblastoma continues to be temozolomide. During adjuvant therapy, rural patients may participate in shared care arrangements, using a combination of local services, visiting oncologists and telehealth consults. Beyond conventional temozolomide and the alternative dose-intensity schedules, salvage options used in Australia include lomustine, bevacizumab, procarbazine, carboplatin and etoposide (11). In addition, patients may be considered for industry-sponsored or investigator-initiated trials when available. Most investigator-initiated national and international trials are coordinated by the Cooperative Trials Group for Neuro-Oncology (COGNO) and conducted across 10 to 20 Australian sites. Alternatively, drugs in early stage development may be accessed via phase I trial networks in the major cities.

Finally, the availability of support services for patients with glioblastoma is highly variable. About $67 \%$ of subspecialty cancer centers have dedicated nurse practitioners and/or brain tumor care coordinators to help patients navigate the complex treatment journey (11). Oncology and palliative care services are increasingly integrated, however resources remain limited. For instance, young patients with significant neurologic disability may be ineligible for admission to a specialist palliative care unit if their prognosis is beyond 3 months, but also ineligible for high-level residential care if they are younger than minimum age requirements. Thus, they become highly dependent on full-time carer support at home. Neurological rehabilitation, neuropsychology and psychiatry are in limited supply and often need to be self-funded by patients.

Education days, support forums and information resources for patients and carers are organized through governmentfunded Australian Cancer Councils in each state or territory. Recently, these information resources have been translated into the ten most common languages spoken in Australia, other than English, to benefit Culturally and Linguistically Diverse communities. There is also substantial advocacy from philanthropic groups including Brain Tumor Alliance Australia (BTAA), the Brain Cancer Group (TBCG), Cure Brain Cancer Foundation (CBCF) and New South Wales Neuro-Oncology Group (NNOG).

\section{Insurance landscape}

Healthcare in Australia is universal and supported by the taxpayer-funded national insurance scheme, Medicare (17).
Medicare covers all inpatient treatment costs in public hospitals. Medicare also rebates outpatient consultation, pathology and radiology services which have been recommended by an independent expert body called the Medical Services Advisory Committee and included in the Medicare Benefits Schedule (MBS) (18). Overall, the proficient and timely clinical management of glioblastoma is well covered by this scheme.

Shortcomings include restricted access to imaging and molecular diagnostics. For instance, fluoro-ethyl-tyrosine (FET) positron emission tomography (PET) scans, and testing for isocitrate dehydrogenase (IDH) mutations, 1p/19q codeletion, MGMT promoter methylation, epidermal growth factor receptor (EGFR) amplification and neurotrophic tyrosine receptor kinase (NTRK) fusions need to be self-funded by patients or absorbed by health services. This is despite the vital importance of IDH mutation and $1 \mathrm{p} / 19 \mathrm{q}$ codeletion status to diagnose glioma subtypes in the revised World Health Organization classification (19).

Other shortcomings include lengthy waiting times to access ancillary services such as neurological rehabilitation, neuropsychology and psychiatry. There are stringent criteria to qualify for social and community disability support services.

Some issues are mitigated with private health insurance. Ancillary and support services can be accessed expeditiously in the private sector. Patients may elect to have treatment under the care of their own specialist in a private hospital. Currently, $44.1 \%$ of Australians pay to have private health insurance (20), although insurance is not comprehensive and significant out-of-pocket costs are accrued by those opting for treatment in the private sector.

Medicines can be reimbursed by the Pharmaceutical Benefits Scheme (PBS) (21). They must first be approved for use in Australia by the Therapeutic Goods Administration (TGA) based on safety and efficacy data, then recommended for use in glioblastoma by an independent expert body, the Pharmaceutical Benefits Advisory Committee (PBAC), based on comparative cost-effectiveness. After a positive PBAC recommendation, medicines are subsequently approved to be PBS-listed. Patients are charged a small copayment fee per prescription, but the substantive cost is borne by government. As such, medicines that are PBSlisted become highly accessible and predominate in local management guidelines. The PBS approval process is lengthy, often lasting months to years, and many medicines are not recommended due to prohibitive cost.

Of relevance here is that temozolomide has been PBS- 
listed since 2005. Bevacizumab has been PBS-listed since August 2019 after a prolonged negotiation process and is now accessible. Carmustine wafer implants $\left(\right.$ Gliadel $\left.^{\circledR}\right)$ are also PBS-listed for glioblastoma, but not in conjunction with temozolomide, contributing to the low uptake of Gliadel $^{\circledR}$. Lomustine and procarbazine are older medicines which are not reimbursed, and thus incur modest out-ofpocket costs or are provided on a case-by-case basis by local health services. Novel targeted drugs or immunotherapy, which may be considered for the treatment of glioblastoma with actionable targets or high tumor mutational burden, are not reimbursed and are often beyond the financial reach of patients, thus limiting off-trial experimental options.

\section{Clinical trials}

The COGNO has successfully conducted Australian investigator-initiated clinical trials for glioblastoma. COGNO has an excellent track record of meeting accrual targets, bolstered by a cohesive network of clinicians and well-informed patients who are keen to participate in research. Clinical trials in Australia are well supported by: funding opportunities from government agencies and philanthropic groups; established partnerships with laboratories and universities to undertake pre-clinical work, biobanking and biomarker discovery; and streamlined access to investigational drugs which only require clinical trial notification (CTN) instead of investigational new drug (IND) agreements.

To highlight the investigator-initiated clinical trials landscape and capacity in Australia, specific examples include the CABARET, VERTU, ACED and NUTMEG trials. CABARET is a completed COGNO-initiated randomized phase II trial for patients with recurrent glioblastoma (22-24). In Part 1, 122 patients from 18 Australian sites were randomized to receive bevacizumab plus carboplatin versus bevacizumab monotherapy. In Part 2, 48 patients were randomized to continue versus cease bevacizumab at progression. The addition of carboplatin did not improve survival or quality of life outcomes, and continuation of bevacizumab beyond progression also did not improve survival. Other COGNO-led trials which are ongoing or awaiting final analysis include: VERTU (ACTRN12615000407594) which randomized 125 glioblastoma patients across 17 Australian sites to longcourse chemoradiotherapy with or without veliparib [brainpenetrant poly ADP-ribose polymerase (PARP) inhibitor]; ACED (ACTRN12615001072505) which randomized 30 glioma patients across 11 Australian sites to acetazolamide (steroid-sparing agent) versus placebo; and NUTMEG (ACTRN12617000267358) which is accruing 102 elderly glioblastoma patients across 20 Australian sites to shortcourse chemoradiotherapy and adjuvant temozolomide with or without nivolumab.

In addition to national cooperative trials, Australia is pioneering the pre-clinical and early phase development of a number of potential targeted drugs for glioblastoma. ACT001 is a brain-penetrant $\mathrm{NF}-\kappa \mathrm{B}$ inhibitor (ACTRN12616000228482). Ifabotuzumab is a monoclonal antibody against receptor tyrosine kinase EphA3 (NCT03374943). ABBV-321 is a $3^{\text {rd }}$ generation antibodydrug conjugate for EGFR-amplified glioblastoma, which occurs in approximately $40 \%$ of cases, and builds upon its predecessor depatuxizumab mafodotin (NCT03234712). Seviteronel is a brain-penetrant P450 C17,20-lyase and androgen receptor (AR) inhibitor for AR-overexpressed glioblastoma, which occurs in approximately $30 \%$ of cases (NCT03600467).

\section{Future directions}

In October 2017, the Australian Minister for Health announced the Australian Brain Cancer Mission (ABCM), a partnership between government and philanthropy which has committed over AUD\$120M to brain cancer research over the upcoming decade (25). Funding flowing from $\mathrm{ABCM}$ has already initiated development of new treatment and imaging clinical trials in glioblastoma. For example, an upcoming COGNO-led adaptive platform trial called MultiArm Glioblastoma Australasia (MAGMA) will test novel variations in the first-line treatment regimen of glioblastoma. In addition to local efforts, COGNO has established an International Collaborative Research Subcommittee (ICRS) to identify and bring promising international trials into Australia, to enable access for our patients.

There is advocacy from clinician and consumer groups to support dedicated nurse practitioners and cancer care coordinators for patients with brain cancer, and to increase government funding for ancillary and support services as part of comprehensive multidisciplinary care. There are efforts to grow national infrastructure for translational neuro-oncology research, including centers of neuropathology and neuro-radiology excellence, a brain cancer rapid autopsy program, and embedded biobanking in neurosurgical units.

Together, we envisage that concerted efforts to improve 
public access to holistic multidisciplinary brain cancer care, and additional investment in our outstanding research programs will finally translate to improvements in glioblastoma outcomes.

\section{Acknowledgments}

Funding: The authors acknowledge support for this work from the Cooperative Trials Group for Neuro-Oncology (COGNO), which receives funding from the Australian Government through Cancer Australia.

\section{Footnote}

Provenance and Peer Review: This article was commissioned by the Guest Editor (Rimas V. Lukas) for the series "The Evolving Landscape of the Management of Glioblastoma" published in Chinese Clinical Oncology. The article did not undergo external peer review.

Conflicts of Interest: All authors have completed the ICMJE uniform disclosure form (available at http://dx.doi. org/10.21037/cco.2020.02.05). HWS: Research funding: AbbVie-payment to institution; Bristol-Myers Squibbpayment to institution. AKN: Consultant or advisory positions (with honoraria): Atara Biotherapeutics-payment to institution; Bayer; Boehringer Ingelheim; Douglas Pharmaceuticals-payment to institution; Merck Sharp Dohme; PharmAbcine; Roche; Trizell Ltd.; Research funding: AstraZeneca-payment to institution; Douglas Pharmaceuticals-payment to institution; Travel funding: AstraZeneca; Boehringer Ingelheim. ZL: Consultant or advisory positions (with honoraria): AbbVie; AstraZeneca; Merck Sharp Dohme; Travel funding: Boehringer Ingelheim; Bristol-Myers Squibb; Merck Sharp Dohme. MK: Consultant or advisory positions (with honoraria): AbbVie; Bristol-Myers Squibb; Eli Lilly; Ipsen; Pfizer; Roche; Research funding: AbbVie-payment to institution; Bristol-Myers Squibb-payment to institution; Specialized Therapeutics-payment to institution.

Ethical Statement: The authors are accountable for all aspects of the work in ensuring that questions related to the accuracy or integrity of any part of the work are appropriately investigated and resolved.

Open Access Statement: This is an Open Access article distributed in accordance with the Creative Commons
Attribution-NonCommercial-NoDerivs 4.0 International License (CC BY-NC-ND 4.0), which permits the noncommercial replication and distribution of the article with the strict proviso that no changes or edits are made and the original work is properly cited (including links to both the formal publication through the relevant DOI and the license). See: https://creativecommons.org/licenses/by-nc$\mathrm{nd} / 4.0 /$.

\section{References}

1. McConigley R, Halkett G, Lobb E, et al. Caring for someone with high-grade glioma: a time of rapid change for caregivers. Palliat Med 2010;24:473-9.

2. Australian Institute of Health and Welfare. Cancer in Australia 2019. Cancer series no 119. Cat no CAN 123. Canberra: AIHW, 2019.

3. Australian Institute of Health and Welfare. Brain and other central nervous system cancers. Cat no CAN 106. Canberra: AIHW, 2017.

4. GBD 2016 Brain and Other CNS Cancer Collaborators. Global, regional, and national burden of brain and other CNS cancer, 1990-2016: a systematic analysis for the Global Burden of Disease Study 2016. Lancet Neurol 2019;18:376-93.

5. Ostrom QT, Cioffi G, Gittleman H, et al. CBTRUS statistical report: primary brain and other central nervous system tumors diagnosed in the United States in 20122016. Neuro Oncol 2019;21:v1-100.

6. Braganza MZ, Kitahara CM, Berrington de González A, et al. Ionizing radiation and the risk of brain and central nervous system tumors: a systematic review. Neuro Oncol 2012;14:1316-24.

7. Bondy ML, Lustbader ED, Buffler PA, et al. Genetic epidemiology of childhood brain tumors. Genet Epidemiol 1991;8:253-67.

8. Australian Bureau of Statistics. Regional population growth, Australia, 2017-18. Cat no 3218.0. Canberra: ABS, 2019.

9. Jong KE, Smith DP, Yu XQ, et al. Remoteness of residence and survival from cancer in New South Wales. Med J Aust 2004;180:618-22.

10. Cramb SM, Mengersen KL, Baade PD. Atlas of cancer in Queensland: Geographical variation in incidence and survival, 1998 to 2007. Brisbane: Viertel Centre for Research in Cancer Control, Cancer Council Queensland, 2011.

11. Chen JY, Hovey E, Rosenthal M, et al. Neuro-oncology practices in Australia: a Cooperative Group for NeuroOncology patterns of care study. Asia Pac J Clin Oncol 
2014;10:162-7.

12. Australian Government Department of Health. Pharmaceutical benefits scheme and repatriation pharmaceutical benefits scheme section 85 supply data. Available online: www.pbs.gov.au

13. Perry JR, Laperriere N, O'Callaghan CJ, et al. Shortcourse radiation plus temozolomide in elderly patients with glioblastoma. N Engl J Med 2017;376:1027-37.

14. Malmström A, Grønberg BH, Marosi C, et al. Temozolomide versus standard 6-week radiotherapy versus hypofractionated radiotherapy in patients older than 60 years with glioblastoma: the Nordic randomised, phase 3 trial. Lancet Oncol 2012;13:916-26.

15. Wick W, Platten M, Meisner C, et al. Temozolomide chemotherapy alone versus radiotherapy alone for malignant astrocytoma in the elderly: the NOA-08 randomised, phase 3 trial. Lancet Oncol 2012;13:707-15.

16. Bernard-Arnoux F, Lamure M, Ducray F, et al. The costeffectiveness of tumor-treating fields therapy in patients with newly diagnosed glioblastoma. Neuro Oncol 2016;18:1129-36.

17. Biggs A. Medicare: a quick guide. Research paper series, 2016-17. Canberra: Parliamentary Library, 2016.

18. Australian Government Department of Health. Medicare benefits schedule. Available online: www.mbsonline.gov.au

Cite this article as: Sim HW, Nowak AK, Lwin Z, Khasraw M. Management of glioblastoma: an Australian perspective. Chin Clin Oncol 2021;10(4):42. doi: 10.21037/cco.2020.02.05
19. Louis DN, Perry A, Reifenberger G, et al. The 2016 World Health Organization classification of tumors of the central nervous system: a summary. Acta Neuropathol 2016;131:803-20.

20. Australian Prudential Regulation Authority. Quarterly private health insurance statistics, Australia, September 2019. Sydney: APRA, 2019.

21. Grove A. The pharmaceutical benefits scheme: a quick guide. Research paper series, 2015-16. Canberra: Parliamentary Library, 2016.

22. Field KM, Simes J, Nowak AK, et al. Randomized phase 2 study of carboplatin and bevacizumab in recurrent glioblastoma. Neuro Oncol 2015;17:1504-13.

23. Field KM, King MT, Simes J, et al. Health-related quality of life outcomes from CABARET: a randomized phase 2 trial of carboplatin and bevacizumab in recurrent glioblastoma. J Neurooncol 2017;133:623-31.

24. Hovey EJ, Field KM, Rosenthal MA, et al. Continuing or ceasing bevacizumab beyond progression in recurrent glioblastoma: an exploratory randomized phase II trial. Neurooncol Pract 2017;4:171-81.

25. Cancer Australia. Australian brain cancer mission. Available online: www.canceraustralia.gov.au/researchdata/research/australian-brain-cancer-mission 